Article

\title{
Distinct Growth and Nutrient Status Responses to Fertilization Regimes in Two Generations of Chinese Fir Seedlings
}

\author{
Ya-Lin Hu ${ }^{1}$, Hui-Hua Fan ${ }^{2}$, Han-Feng Xuan ${ }^{1}$, Abubakari Said Mgelwa ${ }^{1,3}$ and Shi-Pin Chen ${ }^{1, *}$ \\ 1 College of Forestry, Fujian Agriculture and Forestry University, Fuzhou 350002, China \\ 2 Fujian Academy of Forestry Sciences, Fuzhou 350012, China \\ 3 College of Natural Resources Management \& Tourism, Mwalimu Julius K. Nyerere University of Agriculture \\ \& Technology, P.O. Box 976 Musoma, Tanzania \\ * Correspondence: fjcsp@126.com or chenshipin@fafu.edu.cn; Tel.: +86-0591-83781457
}

Received: 1 July 2019; Accepted: 19 August 2019; Published: 21 August 2019

\begin{abstract}
Exponential fertilization is well-known for improving the growth and nutritional status of seedlings during nursery culture. However, less is known about how genetic variability among seedling species influences the effects of fertilization regimes on the growth of seedlings and their nutritional status under varying fertilization levels. Here, we conducted a greenhouse experiment to compare the growth, as well as the nitrogen $(\mathrm{N})$ and phosphorus $(\mathrm{P})$ nutrient concentration responses, of 2- and 3-generation Chinese fir (Cunninghamia lanceolata (Lambert) Hooker) seedlings to the impacts of varying exponential and conventional constant-rate fertilization levels (50, 100, and $200 \mathrm{mg}^{\mathrm{P}}$ seedling ${ }^{-1}$ ). Seedling size and shoot:root ratios were lower in exponential fertilization than in conventional fertilization. Exponentially fertilized seedlings had higher concentrations of $\mathrm{N}$ and $\mathrm{P}$ nutrients compared to conventionally fertilized seedlings during low-level fertilization. Conversely, an elevated $P$ concentration was observed in seedlings subjected to conventional fertilization compared to those exposed to exponential fertilization during high-level fertilization. However, there were no significant differences in seedling $\mathrm{N}$ and $\mathrm{P}$ concentrations between the two fertilization regimes during medium-level fertilization. Furthermore, 2- and 3-generation Chinese fir seedlings differed greatly in their growth and nutritional status. Additionally, there were significant fertilization regime $x$ seedling generation interaction effects on the seedling shoot:root ratio and $N$ concentration. Our findings suggest that exponential fertilization can improve the nutritional status, survival, and growth of Chinese fir seedlings, especially on competitive sites, and that information on both intra-species genetic variability and appropriate fertilizer levels is necessary for the successful fertilization of Chinese fir seedlings, regardless of fertilization regimes.
\end{abstract}

Keywords: Chinese fir seedling; exponential fertilization; fertilization level; genetic variability; seedling nutrients

\section{Introduction}

Nursery stock quality determines the survival and growth rates of out-planted seedlings, thereby subsequently enhancing forest productivity [1]. Consequently, new forestry technologies are continuously evolving globally to improve the quality of seedlings, including their shoot height, root collar diameter (RCD), shoot:root ratio, and internal nutrient pools. Forestry plantations are increasingly shifting towards planting seedlings produced either from high-generation seed orchards through somatic embryogenesis of improved genetic resources [2,3], or steady-state nutrition achieved using an appropriate fertilization technique during nursery culture [1,4]. Because notable intra- or 
interspecies variations in the nutrient use efficiency rate have been documented [5-7], it is necessary to clearly understand the responses of the nutritional status of different plant species to fertilizer loading from fertilization regimes. The relationship between fertilizer dose and seedling internal nutrient concentrations has been well-conceptualized, illustrating nutrient deficiency, luxury consumption, and toxicity in seedlings with increasing fertilizer additions [8,9]. Exponential nutrient loading is a new fertilization technique that has been widely applied to induce steady-state luxury nutrient consumption in seedlings by fertilizing them at exponential rather than conventional (constant) rates, thereby providing nutrient concentrations that more closely match plant requirements during their exponential growth phase [8,10-14]. Seedling morphological and physiological characteristics have revealed varied responses among species to fertilizer loading via an exponential fertilization regime. For example, no considerable differences were noted between exponential fertilization and conventional fertilization in seedling height, biomass, and internal nutrient concentrations in western hemlock (Tsuga heterophylla (Raf.) Sarg.) [15]. On the other hand, exponential fertilization enhanced the N and P concentrations of different tree species such as black spruce (Picea mariana (Mill.) B.S.P.), white spruce (Picea glauca (Moench) Voss), pedunculate oak (Quercus robur L.), Chinese fir (Cunninghamia lanceolata (Lambert) Hooker), and others $[4,8,11,14,16]$. The impacts of exponential fertilization on the growth of seedlings and their nutritional status have been related to the physiological characteristics of tree species (e.g., determinate or indeterminate growth strategy) [14], as well as fertilizer levels [4,14,17]. However, most of these previous studies have adopted one conventional fertilization rate and/or several exponential fertilization rates $[4,11,15]$. Therefore, further work is still needed to evaluate the impacts of several exponential and conventional fertilization rates on seedling growth and nutrients.

There is usually a great range of genetic variation and phenotypic plasticity within species, which in turn, could lead to differences in their responses to nutrient availability. While some studies have indicated an important role of the genotype $\times$ nutrient interaction on tree growth determination $[7,18,19]$, other studies have found no or weak interactions between these variables [20]. One year after planting Pinus radiata on three low fertility sites, the fertilization $\times$ genotype interaction effect on the height growth of the species was significant at only one site, indicating genetic variability among the investigated genotypes in the nutrient use efficiency across sites [6]. Furthermore, the interaction between genotype and nutrient environment gradually decreased with increasing stand age [21]. Although the impacts of exponential fertilization have been well-studied in many tree species, the influences of the interaction between intra-species genetic variation and exponential fertilization on plant morphological and physiological characteristics are still unclear [17,22]. Therefore, it is necessary to evaluate whether intra-species genetic variation in seedlings affects their responses to an exponential fertilization regime.

Chinese fir (Cunninghamia lanceolata (Lambert) Hooker) is the most important commercial tree species widely distributed in southern China, covering an area of approximately $8.95 \times 10^{6}$ ha [23]. Breeding programs for Chinese fir in China started in the early 1960s to produce high genetic quality seeds [3]. Consequently, in 1983, 2-generation, and in 2005, 3-generation, breeding populations were successfully established, with several researchers working towards improving their root morphological traits and growth rates [24,25]. The effects of exponential fertilization on morphological and nutrient dynamics in Chinese fir seedlings have been studied over the past two decades [11,26,27]. However, to the best of our knowledge, there are no studies comparing the responses of different generations of Chinese fir seedlings to the impacts of varying exponential and conventional constant-rate fertilization levels. Therefore, the main objective of this study was to compare the impacts of exponential and conventional fertilization regimes consisting of three fertilization levels on the growth and nutritional status in two generations of Chinese fir seedlings. We hypothesized that (a) exponential fertilization could enhance $\mathrm{N}$ and $\mathrm{P}$ nutrient reserves in Chinese fir seedlings, and (b) the impacts of exponential fertilization would differ between 2-generation and 3-generation seedlings, and would be related to fertilization levels. 


\section{Materials and Methods}

Chinese fir seeds were collected in November 2016 from 2-generation and 3-generation orchards in Fujian province. These seeds were then stored in dry and cool conditions. On 6th April 2017, the seeds were sown at Fujian Agriculture and Forestry University in a greenhouse in 21 cavity containers with $340 \mathrm{~mL}$ volume cavities filled with a mixture of peat, perlite, and vermiculite $(6: 2: 2, v / v / v)$. Each cavity contained three stratified seeds that germinated in about 2 weeks. After germination, the seedlings were thinned to one seedling per cavity. The containers of seedlings were placed on raised benches at an air temperature of $26-28{ }^{\circ} \mathrm{C}$, humidity of $60 \%-85 \%$, and an extended 18-h photoperiod supplemented with fluorescent lamps at a light intensity of $250 \mu \mathrm{mol} \mathrm{s}{ }^{-1} \mathrm{~m}^{-2}$. The initial weights of the individual containers of seedlings were taken at saturation to determine their mass at container capacity. After this, the containers were re-watered every $2-3$ days to maintain them close to $80 \%$ saturation, thereby ensuring no water runoff or nutrient leaching from the containers. The containers were randomly rotated weekly throughout the experimental period to minimize the edge effects in the greenhouse as much as possible.

Fertilization of Chinese fir seedlings started one week after germination using a 15:30:15 (N-P $\mathrm{P}_{2} \mathrm{O}_{5}-\mathrm{K}_{2} \mathrm{O}$ ) fertilizer (composition: 4.5\% $\mathrm{NO}_{3}{ }^{-}-\mathrm{N}, 6.0 \% \mathrm{NH}_{4}{ }^{+}-\mathrm{N}, 4.5 \%$ Urea-N, $30 \% \mathrm{P}_{2} \mathrm{O}_{5}, 15 \%$ $\mathrm{K}_{2} \mathrm{O}, 0.46 \% \mathrm{Mg}, 0.1 \% \mathrm{Fe}, 0.05 \% \mathrm{Mn}, 0.05 \% \mathrm{Zn}, 0.05 \% \mathrm{Cu}, 0.05 \% \mathrm{~B}$, and $0.0005 \% \mathrm{Mo}$ ). To avoid possible $\mathrm{P}$ deficiency in this experiment, fertilizer characterized by a high $\mathrm{P}$ nutrient content was used. Seedlings were exposed weekly to three contrasting fertilization levels (50, 100, and $200 \mathrm{mg} P$ seedling ${ }^{-1}$ ), following two fertilization regimes (a conventional fertilization regime and exponential fertilization regime) for a 20-week fertilization period. After 20 weeks of fertilization, hardening of seedlings was imposed for the next 4 weeks to induce bud setting by reducing the greenhouse photo period to a short-day length $(8 \mathrm{~h})$, lowering greenhouse temperatures $\left(18-20^{\circ} \mathrm{C}\right)$, and reducing the water content (between $40 \%$ and $50 \%$ of container capacity) $[9,10]$. Fertilization rates were chosen based on the projected seedling growth and nutrient uptake rates to achieve limited, maximum, and luxury consumption seedling nutrient conditions. Calculations of weekly nutrient additions via an exponential fertilization technique followed exponential functions to synchronize fertilizer supply with exponential growth and nutrient uptake [28]:

$$
N_{t}=N s\left(e^{r t}-1\right)-N_{(t-1)}
$$

where $N_{t}$ is the nutrient application at time t (in weeks), $N_{S}$ is the initial seedling nutrient reserve, and $r$ is the relative addition rate. A total of twelve treatments were established in this experiment, including 2 seedling generations (2-generation and 3-generation) $\times 2$ fertilization regimes (conventional and exponential) $\times 3$ fertilization levels (50, 100, and $200 \mathrm{mg} \mathrm{P}$ seedling $^{-1}$ ). Each fertilizer treatment was replicated in five different containers, making a total of 630 seedlings/generation or 105 seedlings/treatment.

Seedlings were harvested four times at week $6,16,20$, and 24 . In each sampling time, three or four seedlings per container were destructively sampled. After harvesting, the shoot height and root collar diameter (RCD) were measured per seedling. Moreover, the seedlings were composited for biomass assessment and chemical analysis by treatment replication. Specifically, the seedlings were separated into leaves, stems, and roots. These seedling components were oven-dried for $48 \mathrm{~h}$ at $60^{\circ} \mathrm{C}$ to determine their biomass $(\mathrm{g})$. Seedling materials were then grounded and wet-digested in a block digester using $\mathrm{H}_{2} \mathrm{SO}_{4}-\mathrm{H}_{2} \mathrm{O}_{2}$ solution. Total tissue $\mathrm{N}$ and $\mathrm{P}$ concentrations $\left(\mathrm{mg} \mathrm{g}^{-1}\right.$ ) were determined using a Discrete Wet Chemistry Analyzer (SmartChem 200, AMS Allinace, Rome, Italy).

The current experiment included two fertilization regimes (R), two seedling generations $(G)$, three fertilization levels $(\mathrm{F})$, and four sampling times $(\mathrm{T})$. Seedling size and nutrient status data were analysed using repeated-measures ANOVA with a linear mixed effects model (R 3.5.1). Fertilization 
regimes, seedling generations, fertilization levels, and sampling times were treated as fixed effects, and seedling containers $(\mathrm{C})$ were treated as random effects. The linear mixed effects model was

$$
\mathrm{Y}_{i j k l m}=\mu+\mathrm{R}_{i}+\mathrm{G}_{j}+\mathrm{F}_{k}+\mathrm{T}_{l}+\mathrm{RG}_{i j}+\mathrm{RF}_{i k}+\mathrm{RT}_{i l}+\mathrm{C}_{m(i j k l)}
$$

Using the model, the main effects of R, G, F, and T and their interactions were determined. The differences in means of the investigated experimental variables across treatments were determined by a post-hoc Tukey's Honestly Significant Difference test. Prior to statistical analyses, data were log transformed to meet the normality and homogeneity of variance model assumptions. The significant level was set at $p<0.05$.

\section{Results}

\subsection{Seedling Shoot Height and Root Collar Diameter}

Periodic shoot height and root collar diameter (RCD) measurements of Chinese fir seedlings during the experiment showed an enhanced growth rate in 3-generation seedlings compared to 2-generation seedlings (Figure 1; Table 1). Exponentially fertilized seedlings had a lower shoot height and RCD than conventionally fertilized seedlings ( $p<0.001$, Table 1 ). After 20 weeks of experiment, the shoot height and RCD in seedlings exposed to exponential fertilization decreased by $20.8 \%$ and $20.7 \%$, respectively, compared to those subjected to conventional fertilization. Furthermore, the shoot height and RCD of both 2- and 3-generation Chinese fir seedlings were consistently affected by fertilization regimes (Table 1). There was no Regime $\times$ Level interaction effect on the seedling $\operatorname{RCD}(p=0.271)$. Moreover, seedling RCD, but not shoot height, differed among the three fertilization levels $(p=0.013$ and 0.406 , respectively).


Figure 1. The height $(\mathbf{a}, \mathbf{b})$ and root collar diameter $(\mathbf{c}, \mathbf{d})$ in 2-generation and 3-generation Chinese fir seedlings under conventional and exponential fertilization regimes at three fertilization levels. RCD, root collar diameter; C50, C100, and C200 indicate conventional fertilization delivering a seasonal total of 50, 100, and $200 \mathrm{mg}$ P per seedling at a constant rate; E50, E100, and 200 indicate exponential fertilization adding a seasonal total of 50, 100, and $200 \mathrm{mg}$ P per seedling. 
Table 1. Analysis of variance (ANOVA) on the effects of fertilization regimes, seedling generations, fertilization levels, sampling times, and their interactions on seedling growth and nutrient concentrations.

\begin{tabular}{cccccccc}
\hline \multirow{2}{*}{ Source of Variation } & \multicolumn{7}{c}{ Level of Significance $(p$ value) } \\
\cline { 2 - 8 } & $d f$ & Height & RCD ${ }^{+}$ & Dry Mass & S/R $\ddagger$ & Seedling N & Seedling P \\
\hline Regime (R) & 1 & $<0.001^{* * *}$ & $<0.001^{* * *}$ & $<0.001^{* * *}$ & $<0.001^{* * *}$ & $0.008^{* *}$ & $0.154 \mathrm{~ns}$ \\
Generation (G) & 1 & $<0.001^{* * *}$ & $<0.001^{* * *}$ & $<0.001^{* * *}$ & $0.436 \mathrm{~ns}$ & $0.040^{*}$ & $0.003^{* *}$ \\
Level (L) & 2 & $0.406^{*} \mathrm{~ns}$ & $0.013^{*}$ & $0.036^{*}$ & $0.389 \mathrm{~ns}$ & $<0.001^{* * *}$ & $<0.001^{* * *}$ \\
Time (T) & 3 & $<0.001^{* * *}$ & $<0.001^{* * *}$ & $<0.001^{* * *}$ & $<0.001^{* * *}$ & $<0.001^{* * *}$ & $<0.001^{* * *}$ \\
$\mathrm{R} \times \mathrm{G}$ & 1 & $0.084_{\mathrm{ns}}$ & $0.744 \mathrm{~ns}$ & $0.996 \mathrm{~ns}$ & $0.045^{*}$ & $0.042^{*}$ & $0.175 \mathrm{~ns}$ \\
$\mathrm{R} \times \mathrm{L}$ & 2 & $0.001^{* * *}$ & $0.271_{\mathrm{ns}}$ & $<0.001^{* * *}$ & $0.035^{*}$ & $0.016^{*}$ & $<0.001^{* * *}$ \\
$\mathrm{R} \times \mathrm{T}$ & 3 & $<0.001^{* * *}$ & $<0.001^{* * *}$ & $<0.001^{* * *}$ & $0.055_{\mathrm{ns}}$ & $<0.001^{* * *}$ & $<0.001^{* * *}$ \\
\hline
\end{tabular}

${ }^{\dagger} \mathrm{RCD}$, root collar diameter; $\ddagger \mathrm{S} / \mathrm{R}$, the ratio of shoot dry mass to root dry mass; ns indicates no significant difference; $*, * *$, and $* *$ indicate a significant level at $p<0.05, p<0.01$, and $p<0.001$, respectively.

\subsection{Dry Mass and Shoot:Root Ratio}

Differences in dry mass were observed between two seedling generations, two fertilization regimes, and three fertilization levels (Table 1). After 24 weeks of experiment, the dry mass of 3-generation seedlings increased by $13 \%$ compared to that of 2-generation seedlings (Figure 2). Furthermore, seedling dry mass was declined in exponential fertilization compared to conventional constant-rate fertilization (Figure 2a,b). However, the differences in seedling dry mass between the two fertilization regimes decreased with increasing fertilizer additions and incubation weeks. Specifically, seedling dry mass decreased by $47 \%$ and $27 \%$ at lower and higher fertilization levels, respectively, in the exponential fertilization regime compared with the conventional fertilization regime. Additionally, there was no interaction effect between fertilization regimes and seedling generations on seedling dry mass $(p=0.996$, Table 1).
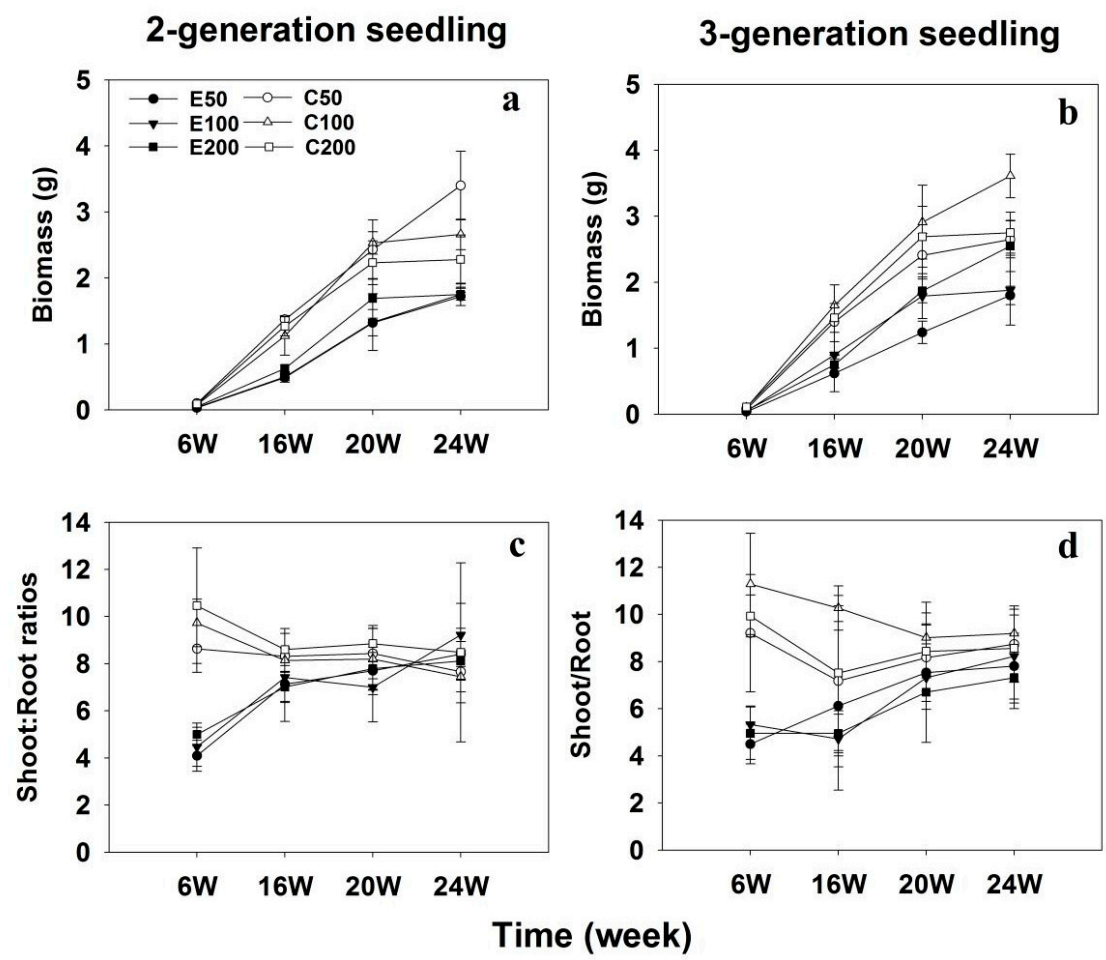

Figure 2. Biomass $(\mathbf{a}, \mathbf{b})$ and shoot:root ratio $(\mathbf{c}, \mathbf{d})$ in 2-generation and 3-generation Chinese fir seedlings under conventional and exponential fertilization regimes at three fertilization levels. C50, C100, and C200 indicate conventional fertilization delivering a seasonal total of 50, 100, and $200 \mathrm{mg} P$ per seedling at a constant rate; E50, E100, and 200 indicate exponential fertilization adding a seasonal total of 50, 100, and $200 \mathrm{mg}$ P per seedling. 
The shoot to root mass ratios of exponentially fertilized seedlings were lower than those of conventionally fertilized seedlings ( $p<0.001$, Table 1 ), but no differences in shoot:root ratios were found between 2- and 3-generation Chinese fir seedlings $(p=0.436)$, and among three fertilization levels $(p=0.389)$. The shoot:root ratios of seedlings subjected to exponential fertilization increased with increasing experimental weeks (Figure $2 c, d$ ), while those of seedlings exposed to conventional fertilization decreased from 6 to 16 weeks of experiment $(p=0.01)$, but revealed no differences between 16,20 , and 24 experimental weeks.

\subsection{Seedling $N$ and $P$ concentrations}

The nitrogen concentration differed between conventionally and exponentially fertilized Chinese fir seedlings ( $p=0.008$, Table 1$)$. The interactions between fertilization regimes and both seedling generations, fertilization levels, and sampling times influenced the seedling $\mathrm{N}$ concentration (all $p<0.05)$. Furthermore, the seedling $\mathrm{N}$ concentration decreased in week $6(-41 \%)$ and $16(-10 \%)$ and increased in week $20(17 \%)$ and $24(13 \%)$ in the exponential fertilization regime compared to the conventional fertilization regime (Table 2). The seedling $\mathrm{N}$ concentration was increased by $13 \%$ in exponential fertilization compared to conventional fertilization under a low fertilization level $(p<0.001$, Figure 3a), but not under medium and high fertilization levels, where no differences in seedling $\mathrm{N}$ concentration were noted between the two fertilization regimes ( $p=0.97$ and 0.51 , respectively). While the $\mathrm{N}$ concentration was higher in exponentially compared with conventionally fertilized 3-generation seedlings $(p<0.001)$, no difference in $\mathrm{N}$ concentration was observed between exponentially and conventionally fertilized 2-generation seedlings $(p=0.65)$.

Table 2. Nitrogen $(\mathrm{N})$ and phosphorus $(\mathrm{P})$ concentrations in 2-generation and 3-generation Chinese fir seedlings under conventional and exponential fertilization regimes with increasing fertilization levels.

\begin{tabular}{|c|c|c|c|c|c|c|c|c|}
\hline \multirow{2}{*}{ Treatment } & \multicolumn{4}{|c|}{ Seedling N Concentration $\left(\mathrm{mg} \mathrm{g}^{-1}\right)$} & \multicolumn{4}{|c|}{ Seedling P Concentration $\left(\mathrm{mg} \mathrm{g}^{-1}\right)$} \\
\hline & Week 6 & Week 16 & Week 20 & Week 24 & Week 6 & Week 16 & Week 20 & Week 24 \\
\hline \multicolumn{9}{|c|}{ 2-generation seedlings } \\
\hline E50 & $1.87 \pm 0.12$ & $18.94 \pm 0.28$ & $20.76 \pm 0.66$ & $23.11 \pm 1.17$ & $0.35 \pm 0.05$ & $0.85 \pm 0.02$ & $2.74 \pm 0.14$ & $2.87 \pm 0.19$ \\
\hline C50 & $3.53 \pm 0.07$ & $19.14 \pm 0.79$ & $17.78 \pm 0.33$ & $19.43 \pm 0.57$ & $0.51 \pm 0.05$ & $0.63 \pm 0.04$ & $2.08 \pm 0.11$ & $2.68 \pm 0.14$ \\
\hline E100 & $2.08 \pm 0.07$ & $20.81 \pm 0.73$ & $23.27 \pm 0.72$ & $27.69 \pm 1.03$ & $0.34 \pm 0.01$ & $0.78 \pm 0.03$ & $3.35 \pm 0.10$ & $3.72 \pm 0.23$ \\
\hline C100 & $3.63 \pm 0.05$ & $25.77 \pm 1.30$ & $21.60 \pm 0.60$ & $26.93 \pm 0.68$ & $0.56 \pm 0.02$ & $0.74 \pm 0.03$ & $2.62 \pm 0.07$ & $3.61 \pm 0.15$ \\
\hline E200 & $3.27 \pm 0.72$ & $23.54 \pm 0.76$ & $26.38 \pm 0.42$ & $36.95 \pm 2.86$ & $0.35 \pm 0.04$ & $0.82 \pm 0.02$ & $3.40 \pm 0.13$ & $5.45 \pm 0.12$ \\
\hline $\mathrm{C} 200$ & $3.98 \pm 0.17$ & $26.25 \pm 1.28$ & $26.13 \pm 0.72$ & $32.31 \pm 1.34$ & $0.61 \pm 0.08$ & $0.98 \pm 0.06$ & $4.20 \pm 0.27$ & $5.80 \pm 0.82$ \\
\hline \multicolumn{9}{|c|}{ 3-generation seedlings } \\
\hline E50 & $1.94 \pm 0.10$ & $18.96 \pm 0.74$ & $22.75 \pm 0.62$ & $27.47 \pm 0.61$ & $0.34 \pm 0.04$ & $0.84 \pm 0.06$ & $3.44 \pm 0.16$ & $4.31 \pm 0.14$ \\
\hline C50 & $3.87 \pm 0.12$ & $20.44 \pm 0.55$ & $15.13 \pm 0.52$ & $21.26 \pm 0.93$ & $0.53 \pm 0.04$ & $0.72 \pm 0.05$ & $2.07 \pm 0.10$ & $3.26 \pm 0.20$ \\
\hline E100 & $2.48 \pm 0.19$ & $18.63 \pm 1.07$ & $22.67 \pm 0.84$ & $29.33 \pm 1.51$ & $0.37 \pm 0.03$ & $0.76 \pm 0.11$ & $3.55 \pm 0.42$ & $4.51 \pm 0.24$ \\
\hline $\mathrm{C} 100$ & $3.87 \pm 0.12$ & $23.65 \pm 1.01$ & $17.79 \pm 1.08$ & $23.84 \pm 0.32$ & $0.65 \pm 0.04$ & $0.81 \pm 0.02$ & $2.74 \pm 0.25$ & $4.33 \pm 0.09$ \\
\hline E200 & $1.96 \pm 0.14$ & $23.98 \pm 1.53$ & $27.03 \pm 0.31$ & $30.70 \pm 1.05$ & $0.30 \pm 0.01$ & $0.73 \pm 0.09$ & $3.73 \pm 0.23$ & $5.05 \pm 0.23$ \\
\hline C200 & $4.34 \pm 0.10$ & $23.02 \pm 0.81$ & $23.76 \pm 0.69$ & $30.97 \pm 1.17$ & $0.71 \pm 0.07$ & $0.74 \pm 0.08$ & $4.06 \pm 0.14$ & $5.42 \pm 0.20$ \\
\hline
\end{tabular}

The values are the means \pm SE $(n=5)$.

Seedling P concentration was not significantly affected by the fertilization regime $(p=0.154$, Table 1). Although there was no Regime $\times$ Generation interaction effect on seedling P concentration $(p=0.175)$, there were Regime $\times$ Level and Regime $\times$ Time interaction effects on seedling $\mathrm{P}$ concentration (all $p<0.001$, Table 1). Seedling P concentration was higher under a low fertilization level $(p<0.001$, Figure $3 b)$ and lower under a high fertilization level $(p=0.02)$ in exponential fertilization compared to conventional fertilization. However, no difference in seedling $\mathrm{P}$ concentration was found under a medium fertilization level between the two fertilization regimes $(p>0.05)$.

Seedling $\mathrm{N}$ and $\mathrm{P}$ concentrations were significantly influenced by fertilization levels and seedling generations (Table 1). The concentrations of $\mathrm{N}$ and $\mathrm{P}$ in Chinese fir seedlings increased with increasing fertilizer additions (Figure 3); specifically, nutrient concentrations in seedlings exposed to high fertilization levels were significantly higher than in those subjected to low fertilization levels. While the $\mathrm{N}$ concentration was greatly enhanced in 2-generation seedlings compared with 3-generation seedlings $(p=0.040$, Table 1$)$, the P concentration was more elevated in 3-generation seedlings than in 2-generation seedlings $(p=0.003)$. 



Figure 3. The effects of two fertilization regimes on (a) nitrogen $(\mathrm{N})$ and $(\mathbf{b}) \mathrm{P}$ nutrient concentrations of seedlings across three fertilization levels. CF, conventional constant rate fertilization; EF, exponential fertilization. * and ${ }^{* * *}$ indicate significant difference at $p<0.05$ and $p<0.001$, respectively; ns indicates no significant difference.

\section{Discussion}

The improvement of internal nutrient reserves during nursery culture is vital in enhancing the survival, growth, and competitive ability of out-planted seedlings [1,4,8,29]. The patterns of reduced seedling growth and increased seedling $\mathrm{N}$ concentration in exponentially compared with conventionally fertilized seedlings have been widely observed for many plant species, including black spruce [1], white spruce [4], western hemlock [15], and trembling aspen [14]. Similarly, this study revealed limited growth, despite the enhanced $\mathrm{N}$ nutrient concentration in Chinese fir seedlings that were exposed to exponential fertilization. However, $\mathrm{Xu}$ and Timmer [11] successfully achieved a steady-state nutrient condition and superior growth performance in Chinese fir seedlings by using the modified exponential fertilization technique that elevated the initially applied fertilizer to compensate for the possible incomplete exploitation of the growing medium by small root systems. Low fertilizer additions during the initial experimental weeks in our study might have limited the growth and nutrient uptake in seedlings under the exponential fertilization regime compared to those under the conventional fertilization regime [14]. This was clearly supported by the increased $\mathrm{N}$ concentration in exponentially fertilized Chinese fir seedlings following high fertilizer additions during both the later stages of fertilization (week 16-20) and hardening period (week 20-24), which in turn reduced the seedling size differences between exponential and conventional fertilization regimes. Exponential fertilization has been playing an increasingly important role in inducing and maintaining seedling steady-state internal nutrient concentrations through the sustainable provision of nutrients that are required by seedlings for their growth and development $[10,15]$.

The relative biomass allocation to roots has been an important and integral indicator for evaluating the quality and performance of stock seedlings $[30,31]$ and seedlings planted on nutrient-poor and/or 
periodically dry sites are often characterized by lower shoot:root ratios [8]. Using Norway spruce and silver birch seedlings, Rytter et al. [32] suggested that increased biomass allocation to roots rather than shoots could be of more importance for future growth and the development of plant height. The results of this study showed a decreased seedling shoot:root ratio in exponential fertilization compared to conventional constant-rate fertilization (Figure $2 \mathrm{c}, \mathrm{d}$ ). The observed relative biomass partitioning toward less shoot production and more root production might enhance the nutrient uptake in exponentially fertilized seedlings with increasing fertilizer additions $[11,14,32,33]$. However, no significant differences in shoot:root ratios were found in western hemlock (Tsuga heterophylla (Raf.) Sarg.) among nutrient treatments [15]. Furthermore, in some studies, early shoot growth was favored under a conventional constant-rate fertilization regime at the expense of root growth, but with much more stable shoot:root balance under an exponential fertilization regime $[4,8]$. In the present study, seedlings subjected to conventional fertilization allocated relatively more biomass to shoots compared to those exposed to exponential fertilization, but with an increasing rather than stable shoot:root trend in the exponential fertilization regime. Our results imply that the responses of seedling morphological features to fertilization regimes are complex, with intra-species variations.

Growth changes among seedling families and/or clones have been largely observed in coniferous and broad-leaved species $[17,34,35]$. Similarly, Chinese fir species have expressed a greater range of heritable variations between families or provenances [3], resulting in larger differences in their growth and nutrient use efficiency [7,36]. For instance, enhanced tree production was observed in 3 -generation compared to 2-generation Chinese fir seedlings [24,37]. Furthermore, the taproot length and lateral root number were increased in 3-generation than in 2-generation Chinese fir seedlings [25]. Consequently, although two breeding generations rather than half-sib families or clones were compared, our results indicate clear variations in the growth and nutrient uptake abilities among these two studied generations of Chinese fir seedlings.

Moreover, we observed significant interactions between seedling generations and fertilization regimes on the seedling shoot:root ratio and $\mathrm{N}$ concentration, but not on seedling size and $\mathrm{P}$ concentration (Table 1). The growth of Chinese fir was significantly affected by the genotype $\times$ environment interaction across four Chinese fir plantation sites in Fujian Province [3]. Because the interaction effects between genetic and environmental nutrient variability on plant morphological and nutritional characteristics are generally complex $[6,17,18,21]$, we suggest that studies to progress understanding of the influences of experimental nutrient loading on seedling internal nutrient pools should take into consideration the large intra-species genetic variations.

Although many previous studies have examined the influences of different fertilization levels under an exponential fertilization regime on seedling morphological characteristics and internal nutrient reserves $[4,9,11,15,38]$, few studies have adopted several fertilization levels using both conventional constant-rate and exponential fertilization regimes. Therefore, the present study was conducted to contribute towards addressing the aforementioned critical knowledge gap. Our results revealed that fertilization levels could alter the impacts of fertilization regimes on seedling growth and nutrient concentrations (Figure 3). While seedling $\mathrm{N}$ and $\mathrm{P}$ concentrations were greatly enhanced under exponential fertilization compared to under conventional constant-rate fertilization during low-level fertilization, no considerable differences in seedling $\mathrm{N}$ and $\mathrm{P}$ concentrations were observed between the two fertilization regimes during medium-level fertilization. Additionally, the P concentration was lower in exponentially compared to conventionally fertilized seedlings during high-level fertilization. Therefore, forest managers should apply appropriate levels of fertilizers to seedlings under both exponential and conventional fertilization regimes to avoid nutrient deficiency or toxicity.

\section{Conclusions}

This study demonstrates that exponential fertilizer loading can lower the growth rate of Chinese fir seedlings, but may improve their internal $\mathrm{N}$ and $\mathrm{P}$ nutrient reserves during nursery culture, especially at low-level fertilization. Consequently, high-quality Chinese fir seedlings were produced with low 
levels of fertilizer under an exponential fertilization regime, thereby supporting the efficient use of fertilizers. Moreover, our findings suggest that seedling genetic variability should be considered when fertilizing seedlings using an exponential fertilization technique. Additionally, the relative biomass partitioning toward more root and less shoot production under an exponential fertilization regime could enhance Chinese fir seedling survival and growth at competitive sites, particularly nutrient-poor sites. However, the survival and growth performance of these seedlings in the field should be evaluated in future studies.

Author Contributions: Formal analysis, H.-H.F.; investigation, H.-F.X.; Writing-Original draft preparation, Y.-L.H.; Writing—Review and editing, A.S.M.; supervision, S.-P.C.

Funding: This research was funded by the National Key R\&D Program of China, grant number 2017YFD0601200 and the International Cooperation Program of Fujian Province, grant number 201710001.

Acknowledgments: We thank Wei Qiang, Liu Zheng, Fu Zhong-Hao, and Huang Zi-Jing for field work and lab analysis.

Conflicts of Interest: The authors declare no conflict of interest. The funders had no role in the design of the study; in the collection, analyses, or interpretation of data; in the writing of the manuscript, or in the decision to publish the results.

\section{References}

1. Timmer, V.R.; Armstrong, G.; Miller, B.D. Steady-state nutrient proconditionning and early outplanting performance of containerized black spruce seedlings. Can. J. For. Res. 1991, 21, 585-594. [CrossRef]

2. Jonsson, A.; Ericsson, T.; Eriksson, G.; Kahr, M.; Lundkvist, K.; Norell, L. Interfamily variation in nitrogen productivity of Pinus sylvestris seedlings. Scand. J. For. Res. 1997, 12, 1-10. [CrossRef]

3. Bian, L.; Shi, J.; Zheng, R.; Chen, J.; Xu, H.X. Genetic parameters and genotype-environment interactions of Chinese fir (Cummnighamia lanceolate) in Fujian Province. Can. J. For. Res. 2014, 44, 582-592. [CrossRef]

4. McAlister, J.A.; Timmer, V.R. Nutrient enrichment of white spruce seedlings during nursery culture and initial plantation establishment. Tree Physiol. 1998, 18, 195-202. [CrossRef]

5. Zvomuya, F.; Rosen, C.J.; Miller, J.C. Response of Russet Norkotah clonal selections to nitrogen fertilization. Am. J. Potato Res. 2002, 79, 231-239. [CrossRef]

6. Zas, R.; Pichel, F.; Martíns, P.; Fernández-López, J. Fertilization $\times$ genotype interaction in Pinus radiata open pollinated families planted in three locations in Galicia (NW Spain). New For. 2006, 32, 253-263. [CrossRef]

7. Wu, P.; Tigabu, M.; Ma, X.; Odén, P.C.; He, Y.; Yu, X.; He, Z. Variations in biomass, nutrition contents and nutrition use efficiency among Chinese fir provenances. Silvae Genet. 2011, 60, 95-105. [CrossRef]

8. Timmer, V.R. Exponential nutrient loading: A new fertilization technique to improve seedling performance on competitive sites. New For. 1996, 13, 275-295.

9. Salifu, K.F.; Timmer, V.R. Optimizing nitrogen loading of Picea mariana seedlings during nursery culture. Can. J. For. Res. 2003, 33, 1287-1294. [CrossRef]

10. Miller, B.D.; Timmer, V.R. Steady-state nutrition of Pinus resinosa seedlings: Response to nutrition loading, irrigation and hardening regimes. Tree Physiol. 1994, 14, 1327-1338. [CrossRef]

11. $\mathrm{Xu}, \mathrm{X}$;; Timmer, V.R. Biomass and nutrition dynamics of Chinese fir seedlings under conventional and exponential fertilization regimes. Plant Soil 1998, 203, 313-322. [CrossRef]

12. Oliet, J.A.; Tejada, M.; Salifu, K.F.; Collazos, A.; Jacobs, D.F. Performance and nutrition dynamics of holm oak (Quercus ilex L.) seedlings in relation to nursery nutrition loading and post-transplant fertility. Eur. J. For. Res. 2009, 128, 253-263. [CrossRef]

13. Isaac, M.E.; Harmand, J.M.; Drevon, J.J. Growth and nitrogen acquisition strategies of Acacia senegal seedlings under exponential phosphorus additions. J. Plant Physiol. 2011, 168, 776-781. [CrossRef]

14. Schott, K.M.; Pinno, B.D.; Landhäusser, S.M. Premature shoot growth termination allows nutrition loading of seedlings with an indeterminate growth strategy. New For. 2013, 44, 635-647. [CrossRef]

15. Hawkins, B.J.; Burgess, D.; Mitchell, A.K. Growth and nutrition dynamics of western hemlock with conventional or exponential greenhouse fertilization and planting in different fertility conditions. Can. J. For. Res. 2005, 35, 1002-1016. [CrossRef] 
16. Quoreshi, A.M.; Timmer, V.R. Early outplanting performance of nutrition-loaded containerized black spruce seedlings inoculated with Laccaria bicolor: A bioassay study. Can. J. For. Res. 2000, 30, 744-752. [CrossRef]

17. Miller, B.D.; Hawkins, B.J. Nitrogen uptake and utilization by slow- and fast-growing families of interior spruce under contrasting fertility regimes. Can. J. For. Res. 2003, 33, 959-966. [CrossRef]

18. Martins, P.; Sampdro, L.; Moreira, X.; Zas, R. Nutritional status and genetic variation in the response to nutrient availability in Pinus pinaster. A multi site field study in Northwest Spain. For. Ecol. Manag. 2009, 258, 1429-1436. [CrossRef]

19. Wanyancha, J.M.; Morgenstern, E.K. Genetic variation in response to nitrogen fertilizer levels in tamarack families. Can. J. For. Res. 1987, 17, 1246-1250. [CrossRef]

20. DeBell, D.S.; Silen, R.R.; Radwan, M.A.; Mandel, N.L. Effect of family and nitrogen fertilizer on growth and foliar nutrients of Douglas-fir seedlings. For. Sci. 1986, 32, 643-652.

21. Gwaze, D.P.; Wolliams, J.A.; Kanowski, P.J.; Bridgwater, F.E. Interactions of genotype with site for height and stem straightness in Pinus taeda in Zimbabwe. Silvae Genet. 2001, 50, 3-4.

22. Zabek, L.M.; Prescott, C.E. Steady-state nutrition of hybrid poplar grownfrom un-rooted cuttings. New For. 2007, 34, 13-23. [CrossRef]

23. Zhang, S. Nonlinear Mixed-Effects Modeling of Variable Exponent Taper Equations with Density for Chinese Fir. Master's Thesis, Chinese Academy of Forestry, Beijing, China, June 2017.

24. Fan, H.; Lai, W.; Huang, Q.; Huang, Y.; Yang, F. Contrast between different superior varieties of Cunninghamia lanceolata at seedling stage. Hubei For. Sci. Technol. 2017, 46, $24-27$.

25. Huang, J.C. Early growth of secondary- and third-generation Chinese firin Tongle forestry centre. Chin. Agric. 2017, 11, 50-51.

26. Xu, X.; Timme, V.R. Growth and nitrogen nutrition of Chinese fir seedlings exposed to nutrition loading and fertilization. Plant Soil 1999, 216, 83-91. [CrossRef]

27. Liu, H.; Wang, C.; Wu, J.; Guo, L. Effects of exponential $\mathrm{N}$ fertilization on the growth and nutrient content in clonal Cunninghamia lanceolata seedlings. Chin. J. Appl. Ecol. 2016, 27, 3123-3128.

28. Ingestad, T.; Lund, A.B. Nitrogen stress in birch seedlings I. growth technique and growth. Physiol. Plant. 1979, 45, 137-148. [CrossRef]

29. Rose, R.; Ketchum, J.S. Interaction of initial seedling diameter, fertilization and weed control on Douglas-fir growth over the first four years after planting. Ann. For. Sci. 2003, 60, 625-635. [CrossRef]

30. Burdett, A.N.; Herring, L.J.; Thompson, C.F. Early growth of planted spruce. Can. J. For. Res. 1984, 14, 644-651. [CrossRef]

31. Grossnickle, S.C. Ecophysiology of Northern Spruce Species: The Performance of Planted Seedlings; NRC Research Press: Ottawa, ON, Canada, 2000; p. 409.

32. Rytter, L.; Ericsson, T.; Rytter, R.M. Effects of demand-driven fertilization on nutrient use, root: Plant ratio and field performance of Betula pendula and Picea abies. Scand. J. For. Res. 2003, 18, 401-415. [CrossRef]

33. Ingestad, T.; Ågren, G.I. The influence of plant nutrition on biomass allocation. Ecol. Appl. 1991, 1, $168-174$. [CrossRef]

34. Huang, H.J.; Peng, R.S.; Liu, Y.; Jiang, J. Growth traits variation analysis and family selection of 3-year-old various ploidy Betula platyphylla. Bull. Bot. Res. 2017, 37, 274-280.

35. Neophytou, C.; Weisser, A.M.; Landwehr, D.; Seho, M.; Kohnle, U.; Ensminger, I.; Wildhagen, H. Assessing the relationship between height growth and molecular genetic variation in Douglas-fir (Pseudotsuga menziesii. provenances. Eur. J. For. Res. 2016, 135, 465-481. [CrossRef]

36. He, P.; Xu, J.; Xu, Y.; Chen, Y.; Shen, F.; Xu, L. Growth differences and selection of Chinese fir families in young plantations with different site conditions. J. Zhejiang A F Univ. 2018, 35, 453-458.

37. Gong, S. The comparison of early growth in the afforestation of different provenance for Chinese fir. Anhui Agric. Sci. Bull. 2015, 21, 85-87.

38. Schmal, J.L.; Jacobs, D.F.; O'Reilly, C. Nitrogen budgeting and quality of exponentially fertilized Quercus robur seedlings in Ireland. Eur. J. For. Res. 2011, 130, 557-567. [CrossRef]

(C) 2019 by the authors. Licensee MDPI, Basel, Switzerland. This article is an open access article distributed under the terms and conditions of the Creative Commons Attribution (CC BY) license (http://creativecommons.org/licenses/by/4.0/). 